\title{
Rapid determination of tetrabromobisphenol A and its main derivatives in aqueous samples by ultrasound-dispersive liquid-liquid microextraction combined with high-performance liquid chromatography
}

\author{
Xuemei Wang ${ }^{\text {a,b }}$, Jiyan Liu ${ }^{\text {a,*, }}$,ian Liu ${ }^{\text {a }}$, Xinzhen Du ${ }^{\text {b }}$, Guibin Jiang ${ }^{\text {a }}$ \\ a State Key Laboratory of Environmental Chemistry and Ecotoxicology, Research Center for Eco-Environmental Sciences, Chinese Academy of Sciences, \\ Beijing 100085, China \\ ${ }^{\mathrm{b}}$ College of Chemistry and Chemical Engineering, Northwest Normal University, Lanzhou 730070, China
}

\section{A R T I C L E I N F O}

\section{Article history:}

Received 3 May 2013

Received in revised form

30 July 2013

Accepted 11 August 2013

Available online 16 August 2013

Keywords:

Dispersive liquid-liquid microextraction

Brominated flame retardants

High-performance liquid chromatography

Ultrasound-assisted method

\begin{abstract}
A B S T R A C T
A method of ultrasound-dispersive liquid-liquid microextraction (US-DLLME) combined with highperformance liquid chromatography/variable wavelength detection (HPLC-VWD) has been developed for rapid measuring tetrabromobisphenol $\mathrm{A}$ and its five derivatives in water. Parameters affecting the extraction efficiency including the extraction solvents and dispersive solvents and their volume, ionic strength of the sample, and ultrasound time were optimized, and further validated by orthogonal array design (OAD). The optimized conditions provided enrichment factors for analytes of 74-490. Most analytes had linear responses between 2 and $500 \mu \mathrm{g} \mathrm{L}{ }^{-1}$, with correlation coefficients $\left(r^{2}\right)$ of $0.9923-$ 0.9994. Limits of detection were $0.13-0.63 \mu \mathrm{g} \mathrm{L}^{-1}$. Relative standard deviations (RSDs) for five replicates ranged from $2.6 \%$ to $4.5 \%$ for all analytes. When applied to spiked samples of real water, the method provided recoveries of $88.6-106.3 \%$ for tap water, $87.8-108.5 \%$ for Mi River water, $82.7-113.5 \%$ for chemical wastewater, $45.5-115.3 \%$ for urine, and $46.4-126.2 \%$ for fruit juice, with RSDs $(n=5)$ less than $4 \%, 6 \%, 8 \%, 10 \%$, and $9 \%$ respectively.
\end{abstract}

(c) 2013 Published by Elsevier B.V.

\section{Introduction}

Environmental and human health problems caused by brominated flame retardants (BFRs) have recently been receiving increased attention [1]. One of the most important BFRs, tetrabromobisphenol A (TBBPA), has the highest production volume, exceeding those of Deca-BDE and HBCD, covering around $60 \%$ of the total BFRs market [2,3], and has been confronted with increasing environmental scrutiny [4].

Available evidence indicates that TBBPA and TBBPA-bis (allyl ether) (TBBPA-BAE) lead to potentially toxic endocrine-disruptor effects, immunotoxicity, and neurotoxicity, all because their structures resemble the thyroid hormone thyroxin (T4) and bisphenol A [5-7]. Other studies have shown that TBBPA can be debrominated under anaerobic and aerobic conditions $[8,9]$ to yield bisphenol A, a widely used compound with a disruptive endocrine function [10]. Therefore, it is urgent to evaluate and monitor the trace levels of these compounds in the environment.

\footnotetext{
* Corresponding author. Tel.: +86 106284 9334; fax: +86 1062849179 .

E-mail address: liujy@rcees.ac.cn (J. Liu).
}

TBBPA has been measured in some water samples. Most of those studies used high-performance liquid chromatography (HPLC) coupled with mass spectrometry (MS) [11,12]. But TBBPA derivatives have received less attention. One study recently measured TBBPA, TBBPA-BAE, and TBBPA-bis (2,3-dibromopropyl ether) (TBBPA-BDBPE) in real samples by LC-MS $\mid$ MS by using accelerated solvent extraction (ASE) as THE sample-extraction method, gel-permeation chromatography (GPC), and solid-phase extraction (SPE) as cleanup technique [13]. Another study proposed pressurized fluid extraction (PFE) and fluidized bed extraction (FBE) as extraction procedures and HPLC-DAD/MS for the determination of TBBPA-BDBPE in environmental samples [14]. However, these extraction methods are time-consuming, tedious and often require large amounts of toxic organic solvent. To overcome those disadvantages, time-saving and high-efficiency microextraction techniques have drawn much attention.

DLLME (dispersive liquid-liquid microextraction) is a new sample pretreatment technique, which is based on a ternary solvent system like homogeneous liquid-liquid extraction and cloud point extraction [15]. In this method, a cloudy solution is formed when an appropriate mixture of a few microliters of organic extraction solvent and a small volume of a dispersive 
solvent are injected into an aqueous sample of the analytes. After centrifuging, the analytes in the sedimentary organic phase can be measured by conventional chromatographic techniques. DLLME is simple, rapid and environmentally friendly, and has been successfully measured trace organic pollutants in environment samples [15-20]. The present work was undertaken to determine TBBPA and its five derivatives by using ultrasound-dispersive liquidliquid microextraction (US-DLLME) coupled with HPLC. To the best of our knowledge, TBBPA and its five derivatives, especially for TBBPA-bis (glycidyl) ether (TBBPA-BGE), have not been measured this way before. This study evaluated the effects of various experimental parameters, and an optimized method was successfully applied to the real water sample analysis.

\section{Experimental section}

\subsection{Materials and chemicals}

Bisphenol A (BPA), TBBPA, TBBPA-BAE, TBBPA-bis (2,3-dibromopropyl ether) (TBBPA-BDBPE), TBBPA-BGE, and TBBP-A bis (2hydroxyethyl ether) (TBBPA-BHEE) were obtained from SigmaAldrich (St. Louis, MO, USA). Their structures are given in the Supporting Information (Table S-1, along with the offical abbreviation [21]). Stock solution of each was prepared at a concentration of $100 \mathrm{mg} \mathrm{L}^{-1}$ in methanol and stored at $4{ }^{\circ} \mathrm{C}$. Working solutions were prepared by appropriately diluting the stock solution with ultrapure water.

Analytical-grade dichloromethane (DCM), chloroform, methanol, acetone, and tetrahydrofuran (THF), and HPLC-grade acetonitrile (ACN) were purchased from J.T. Baker (Phillipsburg, NJ, USA). 1-butyl-3-methylimidazolium hexafluoro phosphate ([BMIM] $\left.\left[\mathrm{PF}_{6}\right]\right)$ (99\%) was purchased from J\&K Chemical Ltd. (Beijing, China), and 1-butyl-3-methylimidazolium bis (trifluoro) ([BMIM] $\left.\left[\mathrm{SF}_{3}\right]\right)$ (99\%) from Adamas Reagent Co. Ltd. (Shanghai, China). Ultrapure water (18.2 M $\Omega$ ) was generated with a Milli-Q (Millipore, Billerica, MA, USA) water purification system.

Samples of natural water and chemical wastewater were collected from the Mi River and local chemical plant (Shouguang, China). Urine samples were provided by volunteers. Fruit juice was bought from supermarket. All samples were filtrated through a $0.45 \mu \mathrm{m}$ glass fiber membrane (Automatic Science, China) and were stored at $4{ }^{\circ} \mathrm{C}$ before being used.

\subsection{Instrumentation}

Chromatographic analysis was performed with an Agilent1100 HPLC system (Agilent Technologies, USA) equipped with a manual injector and variable wavelength detector (VWD). A reverse phase C18 HPLC column (Acclaim120, $5 \mu \mathrm{m}, 150 \mathrm{~mm} \times 4.6 \mathrm{~mm}$, Dionex) was used for separating of the analytes. The mobile phase consisted of $\mathrm{ACN} /$ water, and used at a constant flow of $1.0 \mathrm{~mL} \mathrm{~min}{ }^{-1}$. The initial ratio $(20 \% \mathrm{ACN})$ was held for $3.0 \mathrm{~min}$, then decreased gradually to $0 \%$ at the end of the 12 -min run. The detector wavelength was $214 \mathrm{~nm}$. Microsyringes of 500-1000 $\mu \mathrm{L}$ and 5$50 \mu \mathrm{L}$ (SGE, Australia) were used to inject the extraction solvent and dispersive solvent into the aqueous sample and transfer the sedimentary organic phase into HPLC injector, respectively.

\subsection{Ultrasound-dispersive liquid-liquid microextraction (US- DLLME)}

A scheme of the US-DLLME has been depicted in Fig. 1. A volume of $5.0 \mathrm{~mL}$ water sample was placed in a $10 \mathrm{~mL}$ screw-cap glass tube with conical bottom. To optimize the extraction, a $5.0 \mathrm{~mL}$ water sample spiked with each analyte at $100 \mu \mathrm{g} \mathrm{L}^{-1}$ was used. Then $0.6 \mathrm{~mL}$ of THF (as dispersive solvent) and $25 \mu \mathrm{L}$ of chloroform (as extraction solvent) were rapidly injected into the sample solution. The mixture was gently shaken for $10 \mathrm{~s}$ and ultrasonicated in ultrasonic bath (KQ-300DE, Shanghai, China) for 2 min to form a homogeneous cloudy solution. The phase was separated by a centrifuging at $4000 \mathrm{rpm}$ for $5 \mathrm{~min}$ (DT5-2, Beijing, China). The volume of the sedimentary organic phase was about $15 \mu \mathrm{L}$, from which $10 \mu \mathrm{L}$ was removed for injecting into HPLC. All operations were carried out at room temperature $\left(25^{\circ} \mathrm{C}\right)$.

\subsection{Calculation of enrichment factor (EF)}

In order to evaluate the effect of experimental conditions on the performance of DLLME, an enrichment factor (EF) was calculated [15]:

$\mathrm{EF}=\frac{C_{\mathrm{s}}}{C_{\mathrm{i}}}$

$C_{\mathrm{s}}$, and $C_{\mathrm{i}}$ are the analyte concentration in the sedimentary organic phase and the initial aqueous samples, respectively.

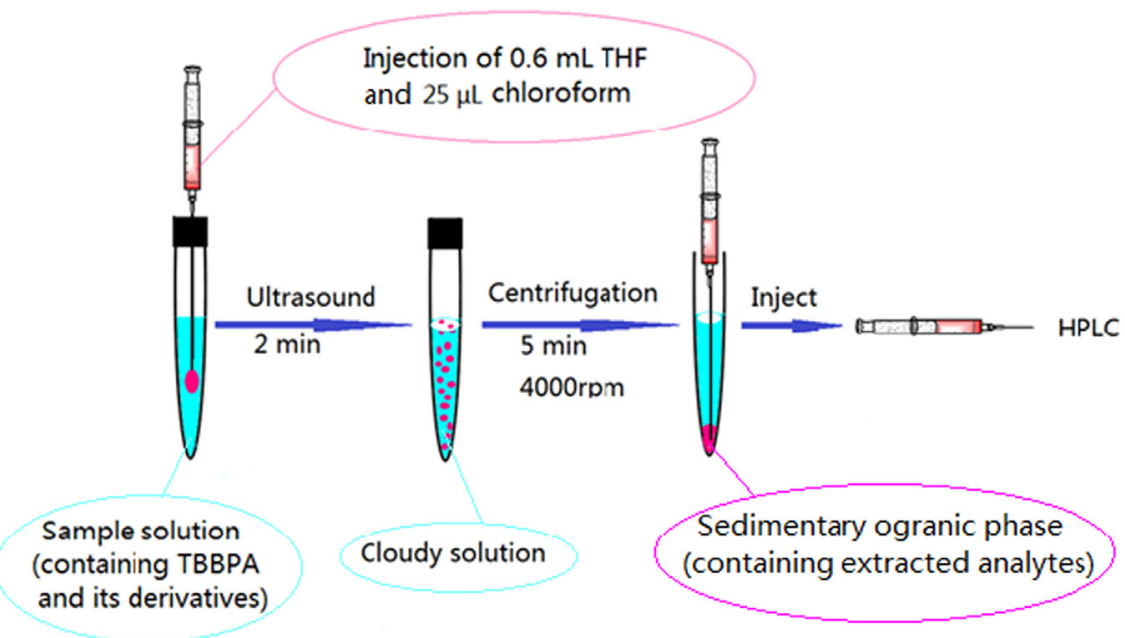

Fig. 1. A scheme of the US-DLLME method. 


\section{Results and discussion}

To maximize extraction efficiency, each factor in the extraction process was optimized. The experiments were performed in triplicate, and the means used.

\subsection{Selection and volume of the extraction solvent}

The choice of extraction solvent is very important for the DLLME process, in order to maximize the extraction efficiency and selectivity for the target compounds. The extraction solvent should be denser than water, behave well during chromatography, extract the target compounds efficiently, has low solubility in water, and be miscible with the dispersive solvent [15]. These considerations led to selecting halogenated alkanes (dichloromethane, chloroform) as an universal extraction solvent and ionic

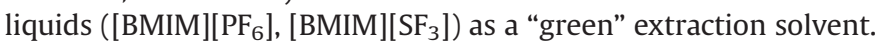
Chloroform was chosen because it gave the highest EFs (Fig. 2a).

This study aimed to determine the minimum volume of extraction solvent necessary to achieve the highest EF. Volumes of chloroform between 10 and $55 \mu \mathrm{L}$ were tested. With less than $10 \mu \mathrm{L}$, centrifuging could not properly separate the phases, and a viscous phase with matrix components remained. When the chloroform volume was increased, an optimum phase separation was observed. The highest EFs were reached with $25 \mu \mathrm{L}$ of chloroform (Fig. 2b). With $10 \mu \mathrm{L}$ of chloroform, lower EFs were observed because the volume was too small to quantitatively extract the BFRs. Increasing the volume of the extraction phase decreased the EF of the analytes, when $C_{0}$ is constant, the sedimentary organic phase volume will increase with the increment of extraction phase volume and the consequent dilution effect of the analyte in sedimentary phase is reduced $C_{\mathrm{s}}$. Therefore, $25 \mu \mathrm{L}$ was selected to separate the phases and provide high enrichment factors.

\subsection{Selection and volume of the dispersive solvent}

A key property of dispersive solvents is miscibility with the extraction solvent and the aqueous sample. For this purpose, methanol, ACN, acetone and THF were investigated. Aqueous samples were extracted in $25 \mu \mathrm{L}$ of chloroform and $0.6 \mathrm{~mL}$ of the dispersive solvent. Dispersive solvents affect the dispersion, stability and equilibrium of the ternary-phase system. An appropriate dispersive solvent will disperse the extraction solvent to fine
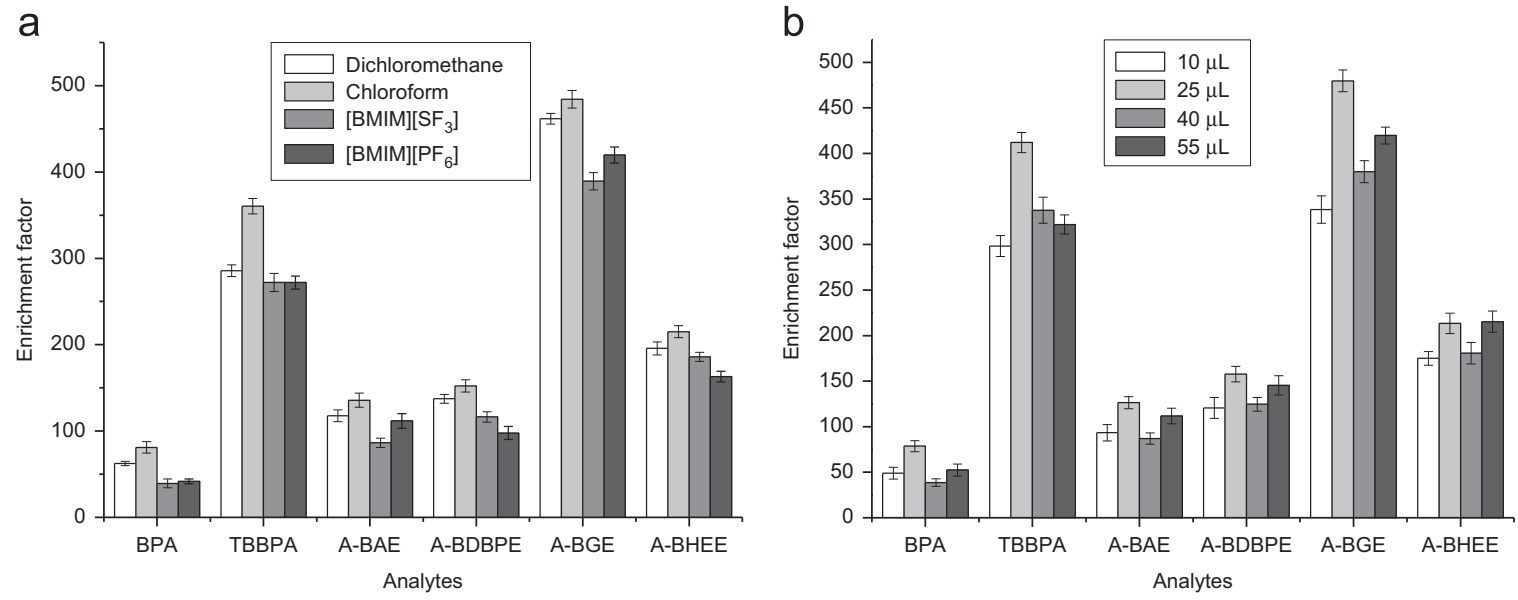

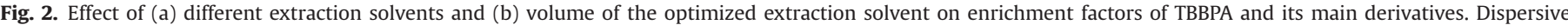
solvent was $0.6 \mathrm{~mL}$ of THF.

a

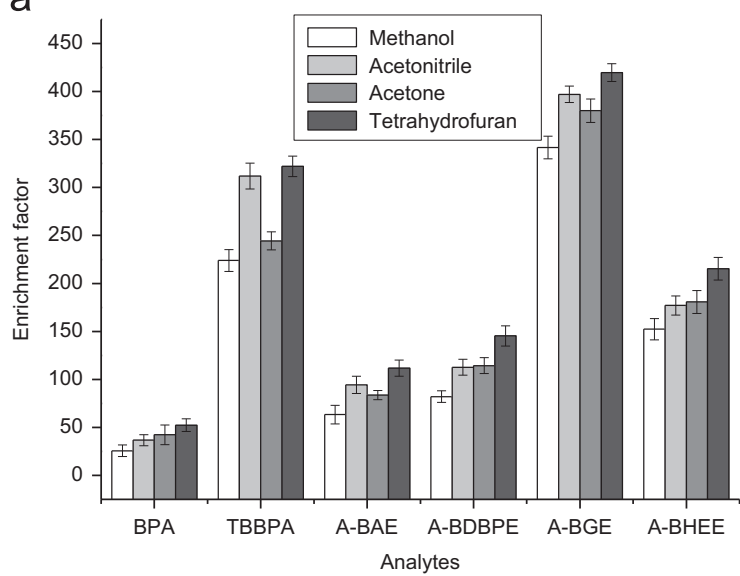

b

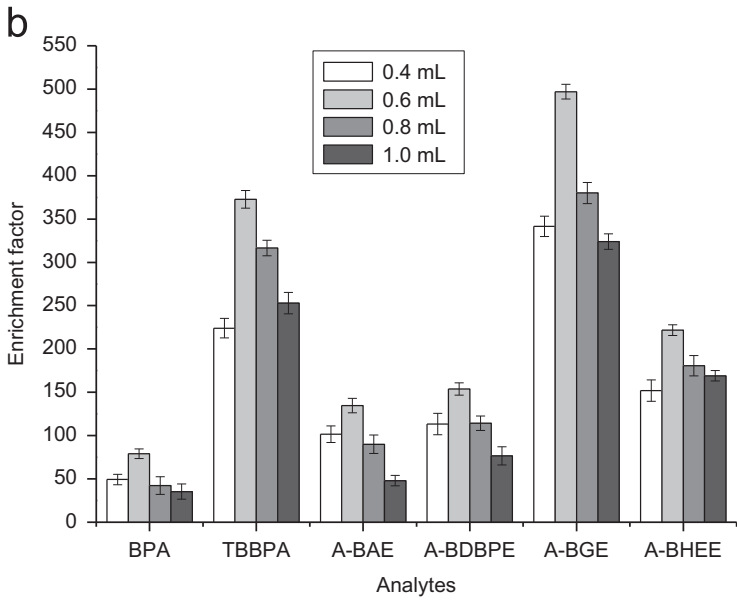

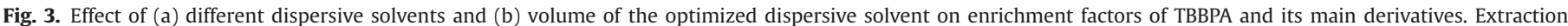
solvent was $25 \mu \mathrm{L}$ of chloroform. 
droplets in water sample and thereby increase the surface area for transferring the target compounds from sample matrix to extraction solvent, and then, the different volumes of sedimentary phase can be obtained after centrifugation. The volumes of sedimentary organic phase obtained with methanol, ACN, acetone and THF were $7,10,8$, and $15 \mu \mathrm{L}$, respectively. THF gave the highest enrichment factor for all the analytes (Fig. 3a), and so was chosen as the dispersive solvent.

The influence of THF volume was tested for $0.4,0.6,0.8$, and $1.0 \mathrm{~mL}$. With increasing volume, the extraction efficiency first increased for all the analytes, then decreased (Fig. 3b). For smaller volumes of THF, a stable cloudy system could not be well formed, resulting in lower enrichments. At higher volumes, the solubility of BFRs in water increased, and led to lower extraction efficiencies. These results led to choosing $0.6 \mathrm{~mL}$ of THF as the optimum.

\subsection{Effect of ionic strength}

Increasing the ionic strength of the sample solution by adding salt usually improves the extraction efficiency in conventional liquid-liquid microextraction via a salting-out effect. Therefore, the effect of ionic strength on US-DLLME was studied by adding up to $20 \%(\mathrm{w} / \mathrm{v}$ ) of $\mathrm{NaCl}$ (Fig. S-1, in Supporting Information).The highest EFs for each target analyte were obtained without $\mathrm{NaCl}$, however. This negative result was attributed to the increase in volume of sedimentary organic phase from 15 to $25 \mu \mathrm{L}$ as a consequence of decreased solubility of the extraction solvent in the aqueous phase after adding the $\mathrm{NaCl}$. Apparently, the dilution dominated the salting-out effect, which was agreement with the results of other researchers [22-24]. Hence, $\mathrm{NaCl}$ was not used.

\subsection{Effect of ultrasonication time}

Ultrasonication can accelerate the homogenization/emulsification step and the formation of the submicrometer droplets that greatly increases the contact surface between liquids and facilitate the transfer of analyte from aqueous sample to organic solvent [15]. To study the effect of ultrasonication time, 0, 2, 5, and $10 \mathrm{~min}$ were tried. The EFs significantly increased up to times of $2 \mathrm{~min}$, then leveled off, suggesting that 2 min was enough to form a stable cloudy solution and complete the extraction. Consequently, 2 min of ultrasonication time was chosen.

\subsection{Orthogonal array design (OAD)}

Orthogonal array design (OAD) was used to confirm the optimal experimental conditions. OAD arranges experiments and analyzes data via orthogonal arrays. $\mathrm{L}_{16}\left(4^{5}\right)$ orthogonal design was used here to find the optimum combination of five influence factors for each target analyte (extraction solvent, dispersive solvent, volume of extraction solvent, volume of dispersive solvent and addition of salt). Each influence factor includes four states (Table S-2 in Supporting Information), which made 16 "Extraction Conditions" in all. Extraction Condition no. 8 was found to be the best (to yield the highest enrichment factors) for all the BFRs except BPA, whose best was Condition no. 4. Thus, Condition no. 8 was adopted for this study, and used in conjunction with sample volumes of $5 \mathrm{~mL}$ and ultrasonication times of 2 min.

\subsection{Performance of the US-DLLME}

In order to validate the developed US-DLLME method, its linearity, correlation coefficients, detection limits, enrichment factors, and repeatability were tested using spiked samples under the optimized conditions. The range of linearity was $2.0-500 \mu \mathrm{g} \mathrm{L}^{-1}$ for most of the target compounds, with the correlation coefficient $\left(r^{2}\right)$ ranging from 0.9923 to 0.9994 (Table 1 ). The limits of detections (LODs) based on a signal-to-noise ratio $(S / N)$ of 3 ranged from 0.13 to $0.63 \mu \mathrm{g} \mathrm{L}^{-1}$. The limits of quantification (LOQs) based on a signalto-noise ratio $(S / N)$ of 10 ranged from 0.43 to $2.10 \mu \mathrm{g} \mathrm{L}^{-1}$. The EFs of BFRs were 74 to 490 . The relative standard deviations (RSDs) for five

Table 1

Performance of US-DLLME

\begin{tabular}{|c|c|c|c|c|c|c|c|}
\hline Analyte & Linear range $\left(\mu \mathrm{g} \mathrm{L}^{-1}\right)$ & $r^{2}$ & $\mathrm{EF}^{\mathrm{a}}$ & $\operatorname{LOD}^{\mathrm{b}}\left(\mu \mathrm{g} \mathrm{L}^{-1}\right)$ & $\mathrm{LOQ}^{\mathrm{C}}\left(\mu \mathrm{g} \mathrm{L}^{-1}\right)$ & $\mathrm{RSD} \%(n=5)$ & Repeatability (RSD\%, day-to-day) \\
\hline BPA & $10-500$ & 0.9985 & 74 & 0.34 & 1.13 & 4.5 & 5.6 \\
\hline ТВBPA & $5.0-500$ & 0.9951 & 356 & 0.13 & 0.43 & 4.3 & 5.3 \\
\hline ТВВРА-ВАE & $2.0-500$ & 0.9923 & 121 & 0.63 & 2.10 & 3.1 & 4.7 \\
\hline TBBPA-BDBPE & $2.0-500$ & 0.9994 & 140 & 0.61 & 2.03 & 2.9 & 6.5 \\
\hline TBBPA-BGE & $2.0-500$ & 0.9956 & 490 & 0.26 & 0.87 & 2.6 & 3.6 \\
\hline TВBPA-BHEE & $10-500$ & 0.9965 & 215 & 0.24 & 0.80 & 3.9 & 4.3 \\
\hline
\end{tabular}

a EF, enrichment factor

${ }^{b}$ LOD, limit of detection for a $S / N=3$.

${ }^{c}$ LOQ, limit of quantification for a $S / N=10$.

Table 2

Comparison of US-DLLME with other sample preparation methods for TBBPA and its derivatives in water.

\begin{tabular}{|c|c|c|c|c|c|c|}
\hline Methods (compounds) & Pre-treatment & Extraction time (min) & LOD & Recovery (\%) & RSD (\%) & Ref. \\
\hline LC-ESI-MS (TBBPA) & Filtration, isotope imprinting & $>90$ & $0.01 \mu \mathrm{g} / \mathrm{L}$ & $>95.0$ & $1.0-4.0$ & {$[11]$} \\
\hline LC-ESI-MS/MS (TBBPA) & SPE & 60 & $0.02 \mu \mathrm{g} / \mathrm{L}$ & $>85.0$ & $5-16$ & [12] \\
\hline LC-APPI-MS/MS (TBBPA and 2 derivatives) & ASE/GPC/SPE & 120 & $0.03-1.28 \mu \mathrm{g} / \mathrm{L}$ & $39-80$ & - & [13] \\
\hline HPLC-DAD/MS (TBBPA-BDBPE) & PFE/FBE & 60 & $10-72 \mu \mathrm{g} / \mathrm{L}$ & $35-98$ & $<2 \%$ & {$[14]$} \\
\hline NACE-PDAD (TBBPA and phenolic compounds) & SPE & $>60$ & $0.8-1.5$ & $73-106$ & $1.9-3.4$ & {$[25]$} \\
\hline LC-ESI-MS/MS (TBBPA) & SPE, filtration & $<20$ & 300 ng g $^{-1}$ (dry weight) & $>95.0$ & - & {$[26]$} \\
\hline HPLC-VWD (TBBPA and 4 derivatives) & SPME & 30 & $0.4-0.9 \mu \mathrm{g} / \mathrm{L}$ & 86.5-103.6 & $1.2-5.1$ & [27] \\
\hline HPLC-VWD (TBBPA and 5 derivatives) & US-DLLME & 2 & $0.13-0.63 \mu \mathrm{g} / \mathrm{L}$ & $82.7-113.5$ & $2.6-4.5$ & This paper \\
\hline
\end{tabular}


replicate runs varied from $2.6 \%$ to $4.5 \%$ at $50 \mu \mathrm{g} \mathrm{L}{ }^{-1}$. The repeatability of day-to-day was 3.6-6.5\% (RSDs). Thus the proposed method has high sensitivity and good repeatability.

The optimized US-DLLME method was compared with other methods for extracting and measuring of TBBPA in water (Table 2).
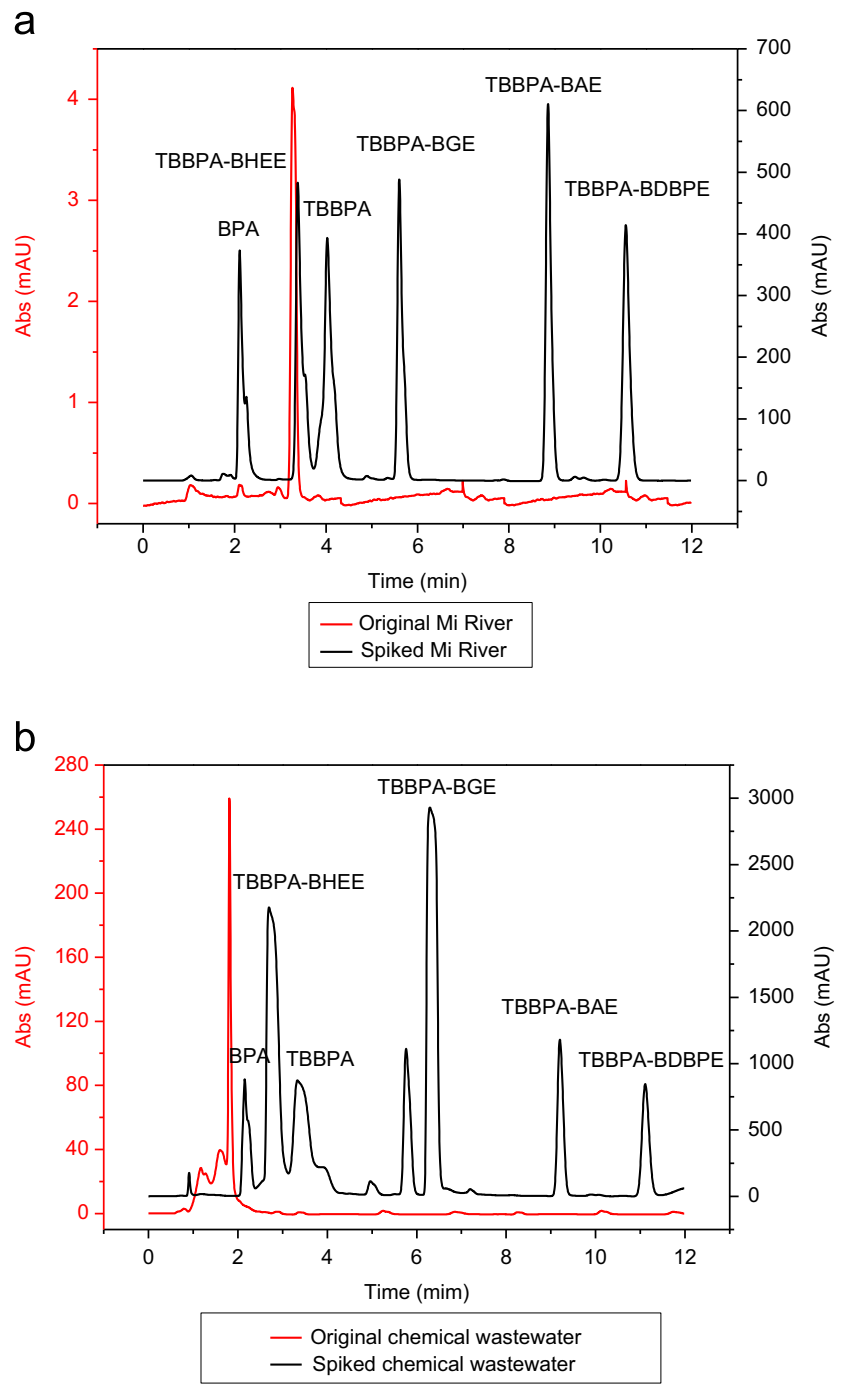

Fig. 4. Chromatogram of (a) the original and spiked Mi River samples (50 $\mu \mathrm{g} \mathrm{L}^{-1}$ for each analyte); (b) the original and spiked wastewater samples $\left(100 \mu \mathrm{g} \mathrm{L}^{-1}\right.$ for each analyte). (1) BPA, (2) TBBPA-BHEE, (3) TBBPA, (4) TBBPA-BGE, (5) TBBPA-BAE, and (6) TBBPA-BDBPE.
Almost all papers have described methods that are only capable of detecting and quantifying TBBPA or one to two derivatives [11-14,25,26]. TBBPA and five derivatives were simultaneously determined in this work only. The extraction time of 2 min was much faster than for traditional methods. The LODs, recoveries and RSDs obtained here are acceptable. Thus, the proposed method is simple, rapid, lowcost, environmentally friendly, and high-throughput.

\subsection{Real water sample analysis}

To investigate the applicability of the optimized US-DLLME method, samples of tap water, the Mi River, wastewater from a chemical plant, urine, and fruit juice were analyzed. To estimate possible matrix influences, aqueous samples were spiked with different concentrations of analytes to determine the recovery of the target analytes. Typical chromatograms for real samples and those spiked with analytes (Fig. 4) showed that several target compounds were found in original aqueous samples. The spiking recoveries were 88.6$106.3 \%$ for tap water, $87.8-108.5 \%$ for the Mi River, $82.7-113.5 \%$ for chemical wastewater, $45.5-115.3 \%$ for urine, and $46.4-126.2 \%$ for fruit juice. The RSDs $(n=5)$ were less than $4 \%, 6 \%, 8 \%, 10 \%$, and $9 \%$ for the five types of samples (Table 3 ). These results showed that the USDLLME method is suitable for measuring BFRs in various aqueous samples, and is not affected significantly by sample matrices.

\section{Conclusions}

A sensitive and simple ultrasound-DLLME method coupled with HPLC-VWD has been developed for the determination of six BFRs in water. The method provides good repeatability, high enrichment factor, and good recovery. In comparison with traditional sample pretreatment methods, such as filtration, SPE, ASE/ GPC/SPE, PFE/FBE, and SPME, the presented method has some obvious advantages in total extraction time (only $2 \mathrm{~min}$ ), cost (instruments and chemicals), green techniques (a small amounts organic solvents). Therefore, the proposed method is rapid, efficient, environmentally friendly and feasible. Since only $5 \mathrm{~mL}$ of sample is needed, the method may be widely applied to different compounds and other aqueous samples such as biological and food samples, although the possible problems posed by their more complex matrices will have to be checked first.

\section{Acknowledgments}

This work was supported by the Natural Science Foundation of China (Nos. 20977096, 21107086, 21265019, and 21107120), the

Table 3

Spiking recoveries and RSDs $(n=5)$ of the BFRs in real water samples.

\begin{tabular}{|c|c|c|c|c|c|c|c|c|c|c|c|c|c|c|c|c|c|c|c|c|}
\hline \multirow[t]{3}{*}{ Analyte } & \multicolumn{4}{|c|}{ Tap water ${ }^{\mathrm{a}, \mathrm{b}}$} & \multicolumn{4}{|c|}{ Mi River ${ }^{\mathrm{a}, \mathrm{b}}$} & \multicolumn{4}{|c|}{ Chemical waste water ${ }^{\mathrm{a}, \mathrm{b}}$} & \multicolumn{4}{|c|}{ Urine sample $e^{a, b}$} & \multicolumn{4}{|c|}{ Fruit juice sample $e^{a, b}$} \\
\hline & \multicolumn{2}{|c|}{ Recovery (\%) } & \multicolumn{2}{|c|}{ RSD (\%) } & \multicolumn{2}{|c|}{ Recovery (\%) } & \multicolumn{2}{|c|}{$\operatorname{RSD}(\%)$} & \multicolumn{2}{|c|}{ Recovery (\%) } & \multicolumn{2}{|c|}{$\operatorname{RSD}(\%)$} & \multicolumn{2}{|c|}{ Recovery (\%) } & \multicolumn{2}{|c|}{ RSD (\%) } & \multicolumn{2}{|c|}{ Recovery (\%) } & \multicolumn{2}{|c|}{$\operatorname{RSD}(\%)$} \\
\hline & $\mathrm{a}$ & $\mathrm{b}$ & $\bar{a}$ & $\mathrm{~b}$ & $\mathrm{a}$ & $\mathrm{b}$ & a & $\mathrm{b}$ & $\mathrm{a}$ & $\mathrm{b}$ & a & $\mathrm{b}$ & a & $\mathrm{b}$ & $\mathrm{a}$ & $\mathrm{b}$ & $\mathrm{a}$ & $\mathrm{b}$ & $\mathrm{a}$ & $\mathrm{b}$ \\
\hline $\mathrm{BPA}$ & 92.5 & 90.6 & 3.8 & 3.5 & 88.7 & 90.3 & 4.1 & 3.4 & 82.7 & 85.5 & 6.7 & 5.6 & 56.7 & 61.2 & 7.3 & 8.1 & 64.1 & 70.2 & 5.4 & 6.1 \\
\hline TBВPA-BHEE & 96.8 & 91.5 & 2.3 & 3.8 & 103.9 & 105.5 & 5.6 & 4.8 & 84.5 & 83.3 & 7.1 & 7.5 & 45.5 & 50.0 & 4.2 & 5.0 & 64.8 & 68.7 & 5.6 & 6.6 \\
\hline TBBPA & 106.3 & 105.4 & 3.1 & 3.0 & 104.8 & 108.5 & 5.1 & 4.6 & 110.9 & 108.7 & 5.3 & 6.3 & 67.1 & 65.5 & 7.6 & 8.5 & 82.9 & 114.2 & 6.5 & 7.1 \\
\hline TВBPA-BGE & 96.2 & 88.6 & 2.2 & 3.5 & 92.8 & 93.5 & 3.6 & 4.7 & 113.5 & 112.2 & 4.9 & 4.8 & 58.6 & 56.5 & 6.4 & 6.2 & 126.2 & 124.5 & 8.8 & 7.6 \\
\hline TBBPA-BAE & 95.4 & 91.0 & 1.8 & 2.6 & 89.2 & 90.1 & 4.5 & 5.5 & 90.1 & 85.8 & 7.3 & 6.6 & 71.1 & 67.8 & 9.5 & 8.8 & 46.4 & 50.2 & 4.7 & 6.5 \\
\hline TВBPA-BDBPE & 102.5 & 93.5 & 2.5 & 2.6 & 87.8 & 89.2 & 3.8 & 4.8 & 83.0 & 86.5 & 6.9 & 7.8 & 115.3 & 110.1 & 7.8 & 8.9 & 123.0 & 119.5 & 9.1 & 8.2 \\
\hline
\end{tabular}

a Spiked with $50 \mu \mathrm{g} \mathrm{L}^{-1}$.

b Spiked with $100 \mu \mathrm{g} \mathrm{L}^{-1}$ 
China Postdoctoral Science Foundation (No. 20110490613), the Key Laboratory of Bioelectrochemistry \& Environmental Analysis of Gansu Province, and the Key Laboratory of Ecological Environment Related Polymer Materials of the Ministry of Education.

\section{Appendix A. Supplementary material}

Supplementary data associated with this article can be found in the online version at http://dx.doi.org/10.1016/j.talanta.2013.08.011.

\section{References}

[1] M. Alaee, P. Arias, A. Sjodin, A. Bergman, Environ. Int. 29 (2003) 683-689.

[2] R.J. Law, C.R. Allchin, J. de Boer, A. Covaci, D. Herzke, P. Lepom, S. Morris, J. Tronczynski, C.A. deWit, Chemosphere 64 (2006) 187-208.

[3] Bromine Science Environmental Forum, http://www.bsef.com (accessed 15.12.2007)

[4] L.S Birnbaum, D.F Staskal, Environ. Health Perspect. 112 (2004) 9-17.

[5] A. Nakajima, D. Saigusa, N. Tetsu, T. Yamakuni, Y. Tomioka, T. Hishinuma, Toxicol. Lett. 189 (2009) 78-83.

[6] A. Covaci, A.C. Gerecke, R.J. Law, S. Voorspoels, M. Kohler, N.V. Heeb, H. Leslie, C.R. Allchin, J.De Boer, Environ. Sci. Technol. 40 (2006) 3679-3688,

[7] G.B Qu, J.B Shi, T. Wang, J.J Fu, Z.N Li, P. Wang, T. Ruan, G.B. Jiang, Environ. Sci. Technol. 45 (2011) 5009-5016.

[8] K.W. George, M.M. Haggblom, Environ. Sci. Technol. 42 (2008) 5555-5561.

[9] Z. Ronen, A. Abeliovich, Appl. Environ. Microbiol. 66 (2000) 2372-2377.
[10] J.W. Voordeckers, D.E. Fennell, K. Jones, M.M. Haggblom, Environ. Sci. Technol. 36 (2002) 696-701.

[11] H. Sambe, K. Hoshina, K. Hosoya, J Haginaka, J. Chromatogr. A 1134 (2006) $16-23$.

[12] G.A. He’ctor, M. Encarnacio’n, T.G. Maria, Rapid Commun. Mass Spectrom. 21 (2007) 4039-4048.

[13] R.J. Letcher, S.G. Chu, Environ. Sci. Technol. 44 (2010) 8615-8621.

[14] R. Köppen, R. Becker, C. Jung, C. Piechotta, I. Nehls, Anal. Bioanal. Chem. 384 (2006) 1485-1492.

[15] M. Rezaee, Y. Assadi, M.M. Hosseini, E. Aghaee, F. Ahmadi, S. Berijani, J. Chromatogr. A 1116 (2006) 1-9.

[16] M. Rezaee, Y. Yamini, S. Shariati, J. Chromatogr. A 1216 (2009) 1511-1514.

[17] Q.H. Wu, C. Wang, Z.M Liu, C.X Wu, X. Zeng, J.L Wen, Z. Wang, J. Chromatogr. A 1216 (2009) 5504-5510.

[18] S.C. Sergiane, P.C. Fabiane, G.P. Ednei, Anal. Chim. Acta 665 (2010) 55-62.

[19] Z.G. Agnieszka, J. Chromatogr. A 1217 (2010) 1761-1766.

[20] M.W Shu, M.I Leong, M.R Fuh, S.D. Huang, Analyst 137 (2012) 2143-2150.

[21] Å. Bergman, A Rydén, R.J. Law, J. de Boer, A. Covaci, M. Alaee, L. Birnbaum, M. Petreas, M. Rose, S. Sakai, N.V. den Eede, I. van der Veen, Environ. Int. 49 (2012) 57-82.

[22] J.X. Wang D.O. Jiang Z.Y. Gu, X.P. Yan, J. Chromatogr. A 1137 (2006) 8-14.

[23] J. Zhou, F. Yang, D. Cha, Z. Zeng, Y. Xu, Talanta 73 (2007) 870-877.

[24] M. Polo, G. Gomez-Noya, J.B. Quintana, M. Llompart, C. Garcia-Jares, R. Cela, Anal. Chem. 76 (2004) 1054-1062.

[25] E. Blanco, M.C. Casais, M.C. Mejuto, R. Cela, J. Chromatogr. A 1071 (1-2) (2005) 205-211.

[26] R. Saint-Louis, E. Pelletiera, Analyst 129 (2004) 724-730.

[27] X.M Wang, J.Y Liu, A.F Liu, Q. Liu, X.Z Du, G.B. Jiang, Anal.Chim. Acta 753 (2012) $1-7$. 